\title{
CDISC SDTM Holter ECG Test Code Terminology
}

National Cancer Institute

\section{Source}

National Cancer Institute. CDISC SDTM Holter ECG Test Code Terminology. NCI

Thesaurus. Code C120523.

Terminology associated with the Holter ECG test code codelist of the Clinical Data

Interchange Standards Consortium (CDISC) Study Data T abulation Model (SDT M). 